\title{
Nonlinear convective pulsation models of type II Cepheids
}

\author{
Radoslaw Smolec \\ Nicolaus Copernicus Astronomical Center \\ ul. Bartycka 18, 00-716 Warszawa, Poland \\ email: smolec@camk.edu.pl
}

\begin{abstract}
We present a grid of nonlinear convective pulsation models of type-II Cepheids: BL Her stars, W Vir stars and RV Tau stars. The models have $0.6 \mathrm{M}_{\odot}$ and cover a wide range of luminosities, effective temperatures and chemical compositions. The most important result is the detection of two domains in the HR diagram, in which period doubling effect is present. First domain, with pulsation periods in between $2 \mathrm{~d}$ and $5.5 \mathrm{~d}$, was also detected in earlier radiative computations. The first star showing the period doubling effect with period in the predicted range $(\approx 2.4 \mathrm{~d})$ was discovered recently. The second domain extends for periods longer than $9.5 \mathrm{~d}$ but so far no period-doubled type-II Cepheid that could be associated with this domain was discovered. We also detect very small domains in which modulation of pulsation is possible. The origin of these phenomena may be assocaited with half-integer resonances between radial pulsation modes. In the most luminous models a dynamical instability is detected, which indicates that pulsation driven mass loss is important process occurring in type-II Cepheids.
\end{abstract}

Keywords. hydrodynamics, stellar oscillations, type-II Cepheids

\section{Introduction}

Type-II Cepheids are low mass, population II stars, pulsating in the radial fundamental mode. Depending on the pulsation period, they are divided into three classes, believed to reflect different evolutionary stages (e.g. Gingold 1985). The shortest period, BL Her variables, have periods between $1 \mathrm{~d}$ and $4 \mathrm{~d}$ and cross the classical instability strip (IS) as they evolve from the blue side of the Zero-Age Horizontal Branch (ZAHB) towards the Asymptotic Giant Branch (AGB). W Vir stars, with periods between $4 \mathrm{~d}$ and $20 \mathrm{~d}$, loop back into the IS due to instabilities in the two shell burning. The longest period RV Tau variables, with periods above $20 \mathrm{~d}$, evolve away from the AGB, crossing the IS on the way to the white dwarf sequence. The characteristic feature of RV Tau stars is period doubling effect. In the light/radial velocity curves we observe alternating deep and shallow minima/maxima; the variation repeats after twice the fundamental mode period. Variability of RV Tau stars becomes more and more irregular as pulsation period is increased. The studies of Buchler, Kolláth \& Serre (1996) and Kolláth et al. (1998) indicate, that deterministic chaos underlies the variability of some RV Tau variables.

The insight into pulsation dynamics of type-II Cepheids is possible thanks to non-linear pulsation modelling. In their radiative models of type-II Cepheids, Kovács \& Buchler (1988) detected not only period doubling, but the full period-doubling route to chaos. In other model sequences the chaos was reached through the tangent bifurcation. Period doubling in their models appears for the first time at periods shorter than observed, at around 10 d. As later analysed by Moskalik \& Buchler (1990) the period doubling in hydrodynamic models may be caused by half-integer resonances between the pulsation modes; in the case of Kovács \& Buchler models the crucial role is played by the 5:2 resonance between the fundamental mode and the second overtone. Period doubling was 
also detected by Buchler \& Moskalik (1992) in their radiative models of BL Her-type stars, with periods between $2 \mathrm{~d}$ and $2.6 \mathrm{~d}$. They traced the origin of the effect to the 3:2 resonance between the fundamental mode and first overtone. As the period doubling effect was robust, the authors concluded that it may finally be detected in the stars. Indeed, 20 years later the first BL Her star with period doubling effect, and pulsation period in the predicted range $(\approx 2.4 \mathrm{~d}$ ) was discovered (Soszyński et al. 2011, Smolec et al. 2012) - a great success of the pulsation theory.

In our recent studies, we have focused our attention on models of BL Her-type, computed with the pulsation code including turbulent convection (Smolec \& Moskalik 2008). These models were characterised by strongly reduced eddy-viscous dissipation, which led to the appearance of extremely interesting dynamical behaviours, quasi-periodic modulation of pulsation, akin to the Blazhko effect observed in RR Lyr stars (Smolec \& Moskalik 2012), or to deterministic chaos with its all flavours, e.g. periodic windows within chaotic bands, intermittency or crises bifurcation (Smolec \& Moskalik 2014). None of these phenomena was observed in BL Her stars yet.

In the present study, in order to investigate which dynamical phenomena persist in more realistic models, we do not reduce the eddy-viscous dissipation. Full results will be reported elsewhere (Smolec, in prep.), here only some results concerning the perioddoubling domains and possible modulation domains are reported.

\section{Models}

All models were computed with the Warsaw convective pulsation codes (Smolec \& Moskalik 2008). All models have $M=0.6 \mathrm{M}_{\odot}$ and adopt three different values of metallicity, $[\mathrm{Fe} / \mathrm{H}]=-1.0,-1.5,-2.0$. In addition, we consider two values of the eddy-viscous dissipation coefficient, $\alpha_{\mathrm{m}}$; in set $\mathrm{A} \alpha_{\mathrm{m}}=0.25$ and such parameter was used in a successful modelling of the only known period doubled BL Her star (Smolec et al. 2012). In set B larger eddy-viscous dissipation is adopted, $\alpha_{\mathrm{m}}=0.5$. Several horizontal model sequences were computed covering the instability strip from $L=50 \mathrm{~L}_{\odot}(\sim \mathrm{RR}$ Lyr domain $)$ up to $L=600 \mathrm{~L}_{\odot}$, with $25 \mathrm{~L}_{\odot}$ step. The computation of more luminous models was not possible due to dynamical instability leading to the decoupling of the outer model zones (to be discussed in more detail in a forthcoming publication). Thus, only the low luminositycool temperature part of the RV Tau domain was reached. Within each sequence, models were computed with $25 \mathrm{~K}$-step in effective temperature. The nonlinear model integration was carried for 2000 pulsation cycles and the resulting time series of radius variation was subject to further analysis.

\section{Results}

Although the majority of our models display single-periodic fundamental mode pulsation, two period doubling domains are identified within the instability strip. They are marked as gray-shaded areas in the HR diagrams plotted in Fig. 1 for the computed model sequences. Darker the area, the more pronounced the alternations. The lower luminosity period doubling domain is present only for models of set A. This domain extends vertically between $150 \mathrm{~L}_{\odot}$ and $425-500 \mathrm{~L}_{\odot}$, and is slightly larger for lower metallicity models. The pulsation periods range from $\approx 2 \mathrm{~d}$ to $\approx 5.5 \mathrm{~d}$ within this domain. It is exactly the same domain as first found by Buchler \& Moskalik (1992) in their radiative models, now revealed in its full extent. The models of Smolec et al. (2012) also fall within this domain. Its origin may be traced to the 3:2 resonance between the fundamental mode and the first overtone as first noted by Buchler \& Moskalik. Loci of this resonance is marked in 

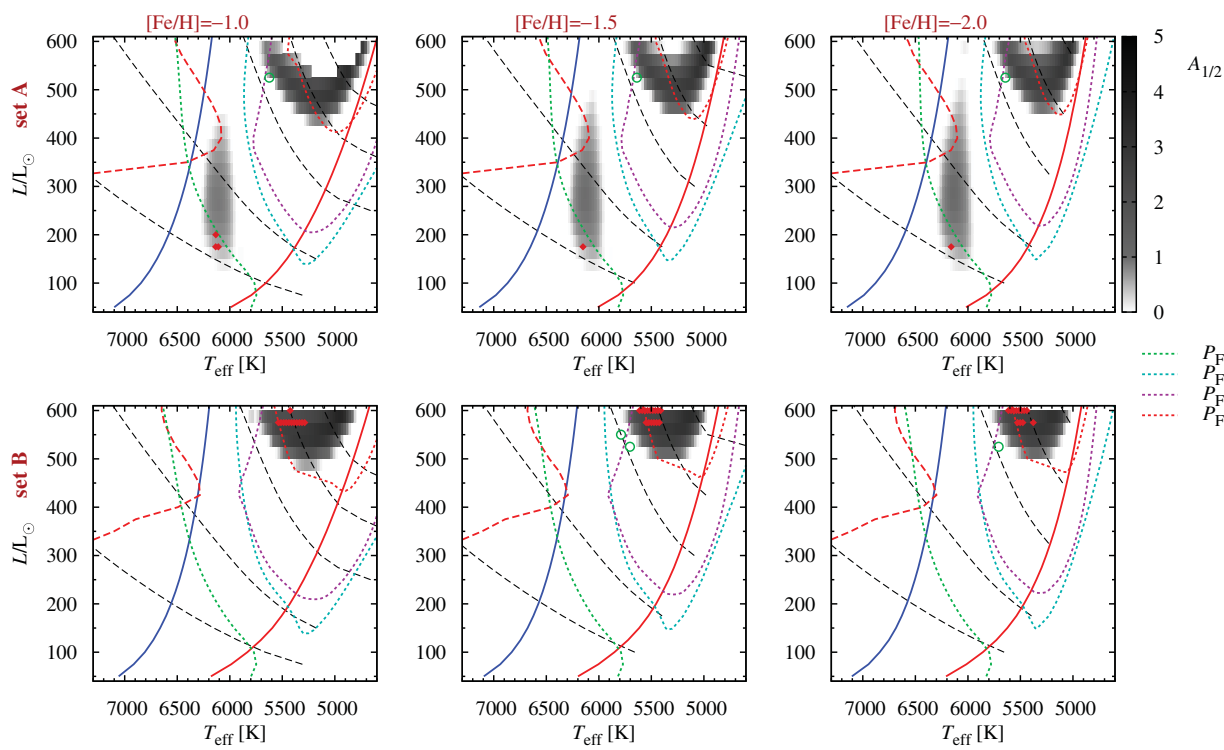

Figure 1. Theoretical HR diagrams showing the location of period doubling domains (gray-shaded areas) for the computed models. Solid blue and red lines mark the edges of classical instability strip. Dashed red line is the red edge of the 4th overtone instability domain. Black dashed lines are lines of constant period (from bottom to top 2, 4, 8, 12, 16 d). Loci of some half-integer resonances are plotted as indicated in the key.

Fig. 1 with green dashed line. Some models within this domain, marked with diamonds in Fig. 1, exhibit period-4 cycle behaviour.

The other period doubling domain extends at higher luminosities, $L>500 \mathrm{~L}_{\odot}$, and is present both for models of set $\mathrm{A}$ and set $\mathrm{B}$. The pulsation periods in this domain start from $\approx 9.5 \mathrm{~d}$. Unfortunately, the full extent of this domain cannot be determined, due to the mentioned instability preventing computation of models more luminous than $\approx 600 \mathrm{~L}_{\odot}$. Within this domain, and for models of set $\mathrm{B}$, period-4 cycle is also detected. Loci of several half-integer resonances run close to the discussed domain (see Fig. 1), and in principle, each may cause the period doubling phenomenon. It seems that the 9:2 resonance with the fourth overtone plays the most important role, as for all period doubled models the mismatch parameter, $\Delta=\omega_{40} / \omega_{\mathrm{F}}-4.5$, is relatively small. In addition fourth overtone is only weakly damped across the period doubling domain. It is a special, surface (or strange) mode, trapped in the outer model layers (see e.g. Buchler \& Kolláth 2001). In fact 4th overtone is unstable on the blue side of the classical instability strip, and also a bit within it. The red edge of the 4 th overtone instability strip is marked with red dashed line in Fig. 1.

None of our models shows period-8 cycle or large amplitude chaotic variations. Such behaviour was detected in the radiative models of Kovács \& Buchler (1988) with the same mass and covering the same luminosity range. Majority of their models however, are cool, located rather far from the blue edge of the instability strip. In fact, as their models are purely radiative, they lack the red edge of the instability strip. The lack of more complex behaviour in the present, convective models, may result from quenching of the pulsation near the red edge of the instability strip. Chaos appears, but only as eddy-viscous dissipation is strongly reduced (Smolec \& Moskalik 2014).

Although large amplitude chaotic variation is not present in the models, in several models, marked with open circles in Fig. 1, we detect periodic modulation of pulsation. 
Computation of additional models reveals the presence of a very narrow ( $\sim 10 \mathrm{~K}$ width) domains in which modulation is present. Modulation periods vary from a few tens to more than $100 \mathrm{~d}$. Interestingly, mean radius is also strongly modulated. It is not easy to point the cause of modulation. We note that half-integer resonances may also cause the modulation of pulsation. Buchler \& Kolláth (2011), based on the solution of appropriate amplitude equations, proposed that the 9:2 resonance between the fundamental mode and the ninth overtone may play a crucial role in the Blazhko effect in RR Lyr stars. Unfortunately, hydrodynamic models of RR Lyr stars do not show the modulation. Period doubling, caused by the 9:2 resonance, is detected in the models (Kolláth, Molnár \& Szabó 2011), but not the modulation. On the other hand, modulation is present in hydrodynamic models of BL Her-type stars with the decreased eddy viscosity (Smolec \& Moskalik 2012). It occurs within period doubling domain (the lower luminosity one) and is also caused by the 3:2 resonance with the first overtone. For the present models, the situation is not that obvious. The models are located very close, but outside the larger luminosity period doubling domain. Although it seems that all modulated models are very close to the loci of the 7:2 resonance with the third overtone, the loci of other half-integer resonances are not far (in the sense of the appropriate mismatch parameter). More detailed analysis will be provided elsewhere.

We note a serious disagreement between the scenario emerging from hydrodynamic models and the observations. The models, both early radiative models and present convective models, predict that period doubling phenomenon should be common among BL Her and W Vir stars. To the contrary, it becomes common only as pulsation period exceeds $20 \mathrm{~d}$, in RV Tau variables. Only one BL Her star shows the effect. Also one W Vir star, the prototype of the class, shows tiny alternations (Templeton \& Henden 2007). Otherwise BL Her and W Vir stars show single-periodic, undoubled oscillations. None type-II Cepheid shows the periodic modulation of pulsation.

\section{Acknowledgement}

This research is supported by the Polish Ministry of Science and Higher Education through Iuventus+ grant (IP2012 036572) awarded to RS. Financial support from the IAU is acknowledged.

\section{References}

Buchler, J. R. \& Moskalik, P., 1992, ApJ, 391, 736

Buchler, J. R. \& Kolláth, Z., 2001, ApJ, 555, 961

Buchler, J. R. \& Kolláth, Z., 2011, ApJ, 731, 24

Buchler, J. R., Kolláth, Z., \& Serre, T., 1996, ApJ, 462, 489

Gingold, R. A., 1985, Mem. Soc. Astron. Ital., 56, 169

Kolláth, Z., Molnár, L., \& Szabó, R., 2011, MNRAS, 414, 1111

Kolláth, Z., Buchler, J. R., Serre, T., \& Mattei, J., 1998, A\&SA, 329, 147

Kovács, G. \& Buchler, J. R., 1988, ApJ, 334, 971

Moskalik, P. \& Buchler, J. R., 1990, ApJ, 355, 590

Smolec, R. \& Moskalik, P., 2008, Acta Astron., 58, 193

Smolec, R. \& Moskalik, P., 2012, MNRAS, 426, 108

Smolec, R. \& Moskalik, P., 2014, MNRAS, 441, 101

Smolec, R., et al., 2012, MNRAS, 419, 2407

Soszyński, I., et al., 2011, Acta Astron., 61, 285

Templeton, M. R. \& Henden, A. A., 2007, AJ, 134, 1999 